\title{
TEA BIOACTIVE COMPOUNDS AS INHIBITOR OF MRSA PENICILLIN BINDING PROTEIN 2a (PBP2a): A MOLECULAR DOCKING STUDY
}

\author{
Marko Jeremia Kalalo ${ }^{\left.1^{*}\right)}$, Fatimawali ${ }^{1)}$, Tekla Kalalo ${ }^{2)}$, Christani Imanuel \\ Jabriel Rambi ${ }^{3)}$ \\ 1)Pharmacy Study Program, Faculty of Mathematics and Natural Sciences, \\ Universitas Sam Ratulangi, Manado 95115, Indonesia \\ 2) Pharmaceutics Department, Faculty of Pharmacy, Universitas Airlangga, \\ Surabaya 60115, Indonesia \\ 3) Apothecary Education Program, School of Pharmacy, Semarang Yayasan Pharmacy \\ Pharmacy College, Semarang 50192, Indonesia \\ *Corresponding Author: markojeremiakalalo@gmail.com
}

\begin{abstract}
Methicillin-Resistant Staphylococcus aureus (MRSA) is a hypervirulent multidrugresistant bacteria. It is spreading around the globe and starting to be a global health problem. It causes bacteremia, infective endocarditis, and bloodstream infection. PBP2a is a protein responsible for MRSA's resistance to antibiotics, especially beta-lactams. Tea contains bioactive compounds such as polyphenols. It is known to have great antibacterial activities. Therefore, this study aims to find potentials antibacterial compounds from tea polyphenolsthat can inhibit PBP2a in MRSA with better binding energy than the currently available drugs using the molecular docking approach. We found that theaflavin $(-9,7$ $\mathrm{kcal} / \mathrm{mol}$ ), as one of the tea polyphenols compound, has a better binding energy with ceftaroline $(9,5 \mathrm{kcal} / \mathrm{mol})$ therefore predicted to have better antibacterial activity. (-)Epigallocatechingallate $(-9,1 \mathrm{kcal} / \mathrm{mol}),(-)$-epicatechingallate $(-8,8 \mathrm{kcal} / \mathrm{mol})$, myricetin $(-8,7 \mathrm{kcal} / \mathrm{mol})$, quercetin $(-8,5 \mathrm{kcal} / \mathrm{mol}),(-)$-epicatechin $(-8,3 \mathrm{kcal} / \mathrm{mol}),(-)$ - epigallocatechin $(-8,3 \mathrm{kcal} / \mathrm{mol})$, kaempferol $(-8,3 \mathrm{kcal} / \mathrm{mol})$, procyanidin $\mathrm{B} 2(-8,1)$, and theflavindigallate $(-7,6 \mathrm{kcal} / \mathrm{mol})$ also have the potential to inhibit MRSA due to its low binding energy.
\end{abstract}

Key words : Molecular docking, MRSA, PBP2a, Tea polyphenols. 


\section{INTRODUCTION}

Methicillin - Resistant Staphylococcus aureus (MRSA) is multidrugresistant Staphylococcus aureus. It is a hypervirulent bacteria that can also be acquired in the community. It is known to spread in the United States years ago. Recently, it has been widespread around the globe. Recent studies have detected MRSA infection in Europe, South America, Asia Pacific (Strauß et al., 2017), Asia, and Africa (Marchello et al., 2020).

MRSA is a pathogen that can cause bacteremia, infective endocarditis, and bloodstream infection. MRSA incidences are found to be increasing in many regions, and it is now one of the leading causes of infective endocarditis in many countries and regions in the world. Strict policies of many countries have regulated the use of antibiotics to treat MRSA. Due to the very few drugs available to treat MRSA, strict policy implementation is applied to maintain its susceptibility (Asgeirsson et al., 2017).

MRSA is hard to treat due to its resistance to all beta-lactam antibiotics. An appropriate resistance mechanism needs to be explored in order to design new potential drugs to treat it. PBP2a is known as a protein responsible for MRSA resistance to antibiotics, especially beta- lactams. It is a protein encoded by a drug- resistant strain contains in a SCCmec box. It shows a low affinity to all beta-lactam antibiotics. To treat MRSA with betalactam antibiotics alone is facing severe challenges and difficulties. Inhibiting PBP2a can reverse its antibiotic resistance and kill the bacteria (Bao et al., 2020).

Medicinal plants are known to possess a great potential as antibacterial drugs (Ogunyemi et al., 2019; Danish et al., 2020; Soyingbe et al., 2018). Tea contains bioactive compounds such as polyphenols. It is known to have great antimicrobial activities. Theaflavin, (-)epigallocatechingallate, (-) -epicatechingallate, myricetin, quercetin, (-)- epicatechin, (-)- epigallocatechin, kaempferol, procyanidin b2, theaflavindigallate are found to be the polyphenols in tea (Yang et al., 2014).

This study aims to find potentials antibacterial compounds from tea polyphenols that can inhibit PBP2a in MRSA with better binding energy than the currently available drugs using molecular docking approach.

\section{MATERIALS AND METHODS Ligands Preparations}

The tea polyphenols known from the previous study (Yang et al., 2014) were searched in Pubchem. The 3D structure was then downloaded with its Pubchem Compound ID was listed in table 1. The ligands' energy was minimized and then converted to autodock PDBQT format using Pyrx.

\section{Receptor Preparations}

PBP2a structured retrieved from Protein Data Bank (PDB : 3ZFZ). The receptor first cleaned from water molecules and its native ligands using Biovia Discovery Studio Visualizer and then optimized using Pyrx.

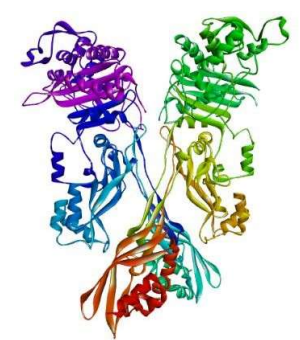

Figure 1. PBP2a (PDB: 3ZFZ) protein structure

\section{Receptor-Ligand docking}

Molecular docking was carried out using Pyrx-vina. Ligands and receptor previously optimized were selected, grid box used for this study are optimized around the active Ser403. Its dimensions were $X: 30 \AA$, Y: $40 \AA, Z: 30 \AA$.

All the processes were carried 
out using a computer with Intel(R) Celeron(R) N4000 CPU @1.10GHz processor, 4Gb RAM, 64-bit operation system.

\section{Analysis and Visualization}

Analysis and visualization of the ligand and receptor interaction waere carried out using Biovia Discovery Studio Visualizer v20.1.0.19295. Both $2 \mathrm{D}$ and $3 \mathrm{D}$ ligand-receptor interaction visualized from molecular docking output file. The output file split first using vina split and command prompt by typing the command: vina_split input (output file name).pdbqt.

The type of bondings was observed in the 2D diagram of the visualization result. Residues involved in the interaction were observed.

\section{RESULTS AND DISCUSSION}

Molecular docking has been widely used in order to design new drugs for a specific purpose. Binding energy result of ligands and MRSA PBP2 presented in Table 1. All tea polyphenols have great binding energy to PBP2a. Molecular docking analyzes molecular interactions between ligands and a specific receptor (Ahmed et al., 2019).

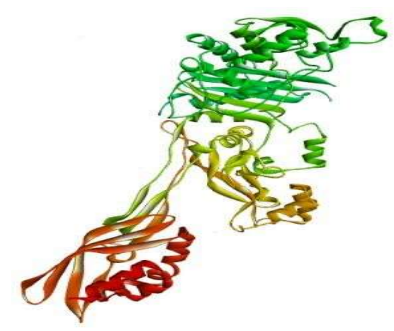

Figure 2. $P B P 2$ a A chain

The protocol was validated by measuring its RMSD value. In this research, the RMSD value of all ligands is $0 \AA$. This value determines two atoms similarity pose. The RMSD from this research suggests the method is valid and can further be used (Kufareva and Abagyan, 2012).

Theaflavin has the best binding energy of all the ligands and control drug ceftaroline. A better ligandreceptor interaction pose was found in theaflavin- PBP2a than ceftaroline PBP2a. It predicted that theaflavin would has a better antibacterial activity against MRSAthan ceftaroline.

Theaflavin is a product of catechin oxidation process. It has significant antibacterial properties against differentbacterial pathogens at varying concentrations. Catechins are found in green tea and black tea, being the major compound of both plants. It has great bioavailability in both urine and plasma suggest it is a potential compound to develop as a drug. Goswami et al. (2020) showed that compounds in black tea inhibited several bacteriahe MIC of black tea extract was found to be $14.72 \mathrm{mg} / \mathrm{ml}$, $3.68 \mathrm{mg} / \mathrm{ml}, 1.42 \mathrm{mg} / \mathrm{ml}, 3.68 \mathrm{mg} / \mathrm{ml}$, and $14.72 \mathrm{mg} / \mathrm{ml}$ against Klebsiella pneumoniae (ATCC 700603), Streptococcus mutans (MTCC 497), Staphylococcus aureus (ATCC 25923), Escherichia coli (ATCC 25922), and Lactobacillus acidophilus (ATCC 43121)respectively.

Table 1. Ceftaroline and tea polyphenols binding energy to PBP2a (PDB: 3ZFZ)

\begin{tabular}{ccc} 
Ligand & $\begin{array}{c}\text { Pubchem } \\
\text { CID }\end{array}$ & $\begin{array}{c}\text { PBP2a } \\
\text { Binding } \\
\text { Energy } \\
\text { (kcal/mol) }\end{array}$ \\
\hline Ceftaroline & 9852981 & $-9,5$ \\
Theaflavin & 114777 & $-9,7$ \\
$\begin{array}{c}(-)- \\
\text { Epigallocatechinga } \\
\text { llate } \\
(-)-\end{array}$ & 65064 & $-9,1$ \\
Epicatechingallate \\
Myricetin \\
$\begin{array}{c}\text { Quercetin } \\
\text { (-)-Epicatechin }\end{array}$ & 5281672 & $-8,7$ \\
$\quad(-)-$ & 5280343 & $-8,5$ \\
Epigallocatechin & 72276 & $-8,3$ \\
Kaempferol & 72277 & $-8,3$ \\
Procyanidin B2 & 5280863 & $-8,3$ \\
Theaflavindigallate & 122738 & $-8,1$ \\
\hline
\end{tabular}




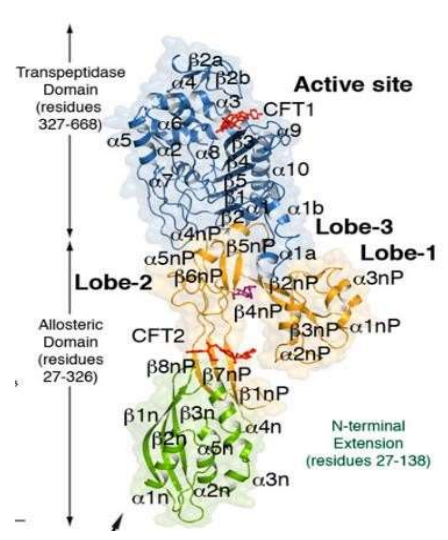

Figure 3. PBP2a active site located at the A chain of the protein inhibitedby ceftaroline (red sticks in bluedomain) in transpeptidase domain (Otero et al., 2013)

PBP2a is responsible for human pathogen $S$. aureus resistance to betalactam class of antibiotics (Alhadrami et al., 2020). Inhibition in the protein's active site leads to deactivation of the protein, making it unable to do a crosslink reaction with peptidoglycan to form a rigid bacteria cell wall. The active site located in the transpeptidase domain suggests that a ligand binds to this active site can stop the cell wall synthesis and further kill the bacteria (Otero et al., 2013). The active site is shown in Figure 3.

$3 \mathrm{D}$ and 2D interactions are shown in Figure 4. and Figure 5. 3D interaction shows both ceftaroline and theaflavin binds to the same pocket of PBP2a. 2D interaction shows amino acids or residues that interact with ligands. Both ceftarolineand theaflavin interact with residues in the transpeptidase domain (residues 327668). It suggests both compounds can bind to PBP2a active site and stop cell wall synthesis.

Theaflavin interacts with the active site by conventional hydrogen bonds, pi-donor hydrogen bonds, pi-pi stack, pi-pi $\mathrm{T}$ shaped, pi-alkyl, unfavorable donor- donor, and unfavorable acceptor-acceptor.

Molecular docking results found to be linear with in-vitro results suggest that ligands with low binding energy with a receptor may have activity using in vitro testing (Cortes et al., 2020).

These results can predict that tea polyphenols can inhibit MRSA by deactivation of PBP2a and stop the cell wall synthesis process.

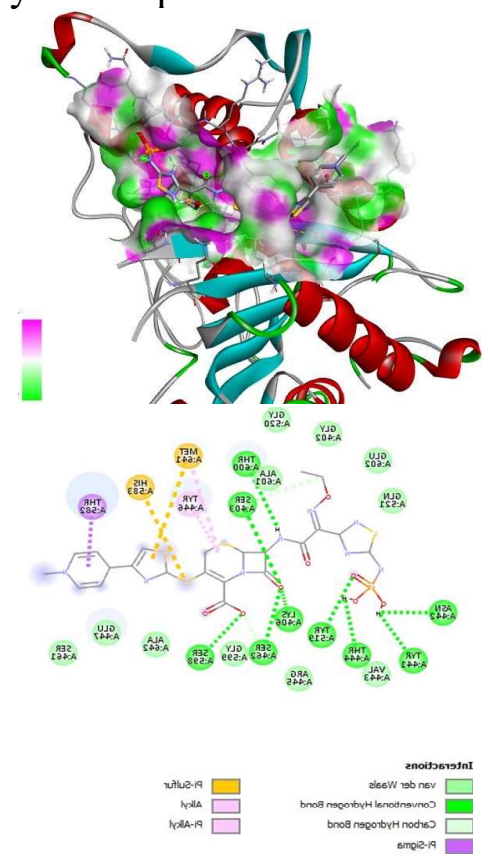

Figure 4. (A) Ceftaroline docking position to PBP2a. (B) 2D diagram interactionof Ceftaroline-PBP2a.

(-) - Epigallocatechingallate, (-)epicatechingallate, myricetin, quercetin, (-) - epicatechin, (-) -epigallocatechin, kaempferol, procyanidin B2, and theflavindigallate also have binding energies lower than $-6 \mathrm{kcal} / \mathrm{mol}$ suggest it have great bindings with PBP $2 \mathrm{a}$ and can be considered a potential compounds to design new antibacterial drug targeting MRSA (Sachdeva et al., 2020).

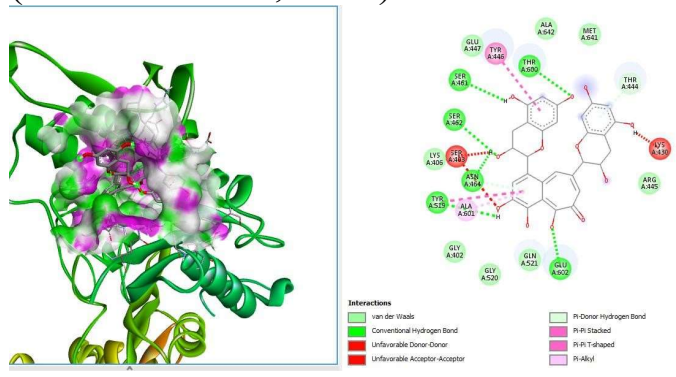

Figure 5. (A) Theaflavin 3D structure dock to PBP2a. (B) Theaflavin-PBP2a interactionin 2D diagram. 


\section{CONCLUSION}

Molecular docking study of this research predicted polyphenols contain in tea can inhibit MRSA by binds to PBP2a active site and stop the bacteria cell wall synthesis. Theaflavin has better binding energy with ceftaroline, therefore predicted to have better antibacterial activity. (-)-Epigallocatechingallate, (-)- epicatechingallate, myricetin, quercetin, (-)-epicatechin, (-)-epigallocatechin, kaempferol, procyanidin $\mathrm{B} 2$, and theflavindigallate also have the potential to inhibit MRSA due to its low binding energy.

\section{REFERENCE}

Ahmed, S., A. Rakib, M. Islam, B. Khanam, F. Faiz, A. Paul, M. Chy, N. Bhuiya, M. Uddin, S. Ullah, M. Rahman, \& T. Emran. 2019. In Vivo and In Vitro Pharmacological Activities of Tacca integrifolia Rhizome and Investigation of Possible Lead Compounds Against Breast Cancer Through in Silico Approaches. Clinical Phytoscience, 5(1).

Alhadrami, H. A., Hamed, A. A., Hassan, H. M., Belbahri, L., Rateb, M. E., \& Sayed, A. M. (2020). Flavonoids as potential anti-MRSA agents through modulation of PBP2A: A computational and experimental study. Antibiotics, 9(9), 1-16.

Asgeirsson, H., Thalme, A., \& Weiland, O. (2017). Staphylococcus aureus bacteraemia and endocarditis epidemiology and outcome: a review Staphylococcus aureus bacteraemia and endocarditis epidemiology and outcome: a review. Infectious Diseases, $0(0)$, 1-18.

Bao, M., Zhang, L., Liu, B., Li, L., Zhang, Y., Zhao, H., Ji, X., Chen, Q., Hu, M., Bai, J., Pang, G., Yi, J., Tan, Y., \& Lu, C. (2020).
Synergistic effects of anti-MRSA herbal extracts combined with antibiotics. Future Microbiology, 15(13), 1265-1276.

Cortes, E., Mora, J., \& Márquez, E. (2020). Modelling the antimethicillin-resistant staphylococcus aureus (MRSA) activity of cannabinoids: A QSAR and docking study. Crystals, 10(8), 120.

Danish, P., Ali, Q., Mm, H., \& Malik, A. (2020). Antifungal And Antibacterial Activity Of Aloe Vera Plant Extract. Biological and Clinical Sciences Research Journal. 1-8.

Goswami, P., \& Kalita, C. (2020). Antibacterial Activity of Black Tea Extract against $\mathrm{S}$. mutans, $\mathrm{S}$. aureus , L . Antibacterial Activity of Black Tea Extract against $\mathrm{S}$. mutans, $S$. aureus, L . acidophilus , Klebsiella and E . coli. J. Evolution Med. Dent. Sci; 9(1):1822.

Kufareva, I. and R. Abagyan. 2012. Methods of protein structure comparison. Methods Mol Biol, 857:231-257.

Marchello, C. S., Dale, A. P., Pisharody, S., Rubach, M. P., \& Crump, A. (2020). crossm A Systematic Review and Metaanalysis of the Prevalence of Community-Onset Bloodstream Infections among Hospitalized Patients in Africa and Asia. 64(1), $1-16$.

Otero, L. H., Rojas-Altuve, A., Llarrull, L. I., Carrasco-López, C., Kumarasiri, M., Lastochkin, E., Fishovitz, J., Dawley, M., Hesek, D., Lee, M., Johnson, J. W., Fisher, J. F., Chang, M., Mobashery, S., \& Hermoso, J. A. (2013). How allosteric control of Staphylococcus aureus penicillin binding protein $2 \mathrm{a}$ enables methicillin resistance and 
physiological function. Proceedings of the National Academy of Sciences of the United States of America, 110(42), 16808-16813.

Oyedemi, B. O. M., Oyedemi, S. O., Swain, S. S., Prieto, J. M., \& Stapleton, P. (2020). Bactericidal and antibiotic-modulation activities of methanol crude extracts of Ligustrum lucidum and Lobelia inflata against MRSA phenotypes: Molecular docking studies of some isolated compounds from both plants against DNA gyrase A. South African Journal of Botany, 130, 54-63.

Sachdeva, C., Wadhwam, A., Kumari, A., Hussain, F., Jha, P., Kaushik, N.K. 2020. In Silico Potential of Approved Antimalarial Drugs for Repurposing Against COVID-19. OMICS A Journal of Integrative Biology. 24(10): 568-580.

Soyingbe, O. S., Mongalo, N. I., \& Makhafola, T. J. (2018). In vitro antibacterial and cytotoxic activity of leaf extracts of Centella asiatica ( $\mathrm{L}$ .) Urb , Warburgia salutaris ( Bertol . F .) Chiov and Curtisia dentata ( Burm . F .) C . A . Sm - medicinal plants used in South Africa. 3, 110.

Strauß, L., Stegger, M., Eberechi, P., Alabi, A., Breurec, S., \& Coombs, G. (2017). Origin, evolution, and global transmission of communityacquired Staphylococcus aureus ST8. 1-9.

Yang, Z. F., Bai, L. P., Huang, W. B., Li, X. Z., Zhao, S. S., Zhong, N. S., \& Jiang, Z. H. (2014). Comparison of in vitro antiviral activity of tea polyphenols against influenza A and $B$ viruses and structure-activity relationship analysis. Fitoterapia, 93, 47-53. 